\title{
A cluster of infectious syphilis cases
}

1. Cobridge Sexual Health Centre, Cobridge, Stoke-on-Trent, 2. Public Health England, West Midlands

\section{Introduction}

In 2016 the Field Epidemiology Service (FES) at Public Health England (PHE) noted an increase in cases of infectious syphilis reported to the Enhanced Syphilis Surveillance Scheme (ESSS) from our clinic in Stoke-on-Trent. From 01 January 2016 to 31 December 2016, 56 cases were reported to ESSS compared with 12 cases from January to December 2015.

\section{Methods}

A confirmed case was defined as any person with clinically compatible symptoms/signs of infectious syphilis (primary, secondary or early latent) with laboratory confirmation using the appropriate combination of non-treponemal and treponemal tests from 1 January 2016 and attending the Cobridge clinic.

Data was extracted from GUMCAD and the ESSS. FES collated and analysed the data using appropriate measures of disease frequency, central tendency and spread in order to describe the epidemiological characteristics of the cluster. All analyses were undertaken in Microsoft Excel.

\section{Results}

Between 01 January 2016 and 31 December 2016, there were 56 cases of syphilis infection diagnosed at the Cobridge Clinic. 21 cases (38\%) were diagnosed as primary syphilis, 20/56 (36\%) were diagnosed as secondary syphilis and $15 / 56(27 \%)$ as early latent syphilis.

All of the cases reported were male and the median age was 37 years (range 16 years to 74 years). 50 of the 56 cases $(89 \%$ ) were of white British ethnicity, $4 / 56(7 \%)$ were of white other ethnicity and $2 / 56(4 \%)$ were of black Caribbean ethnicity.

The majority of cases, $42 / 56,(75 \%)$ reported themselves as being men who have sex with men (MSM), 10/56 (18\%) reported as being heterosexual and 4/56 (7\%) reported being bisexual.

$36 / 56$ cases (64\%) were HIV negative at diagnosis with 16/56 (29\%) HIV positive; HIV status was unknown for $4 / 56$ (7\%) of cases.

Fig 1. Cases of infectious syphilis reported to ESSS by month, Cobridge Clinic, 01 January 2013 to 31 December 2016

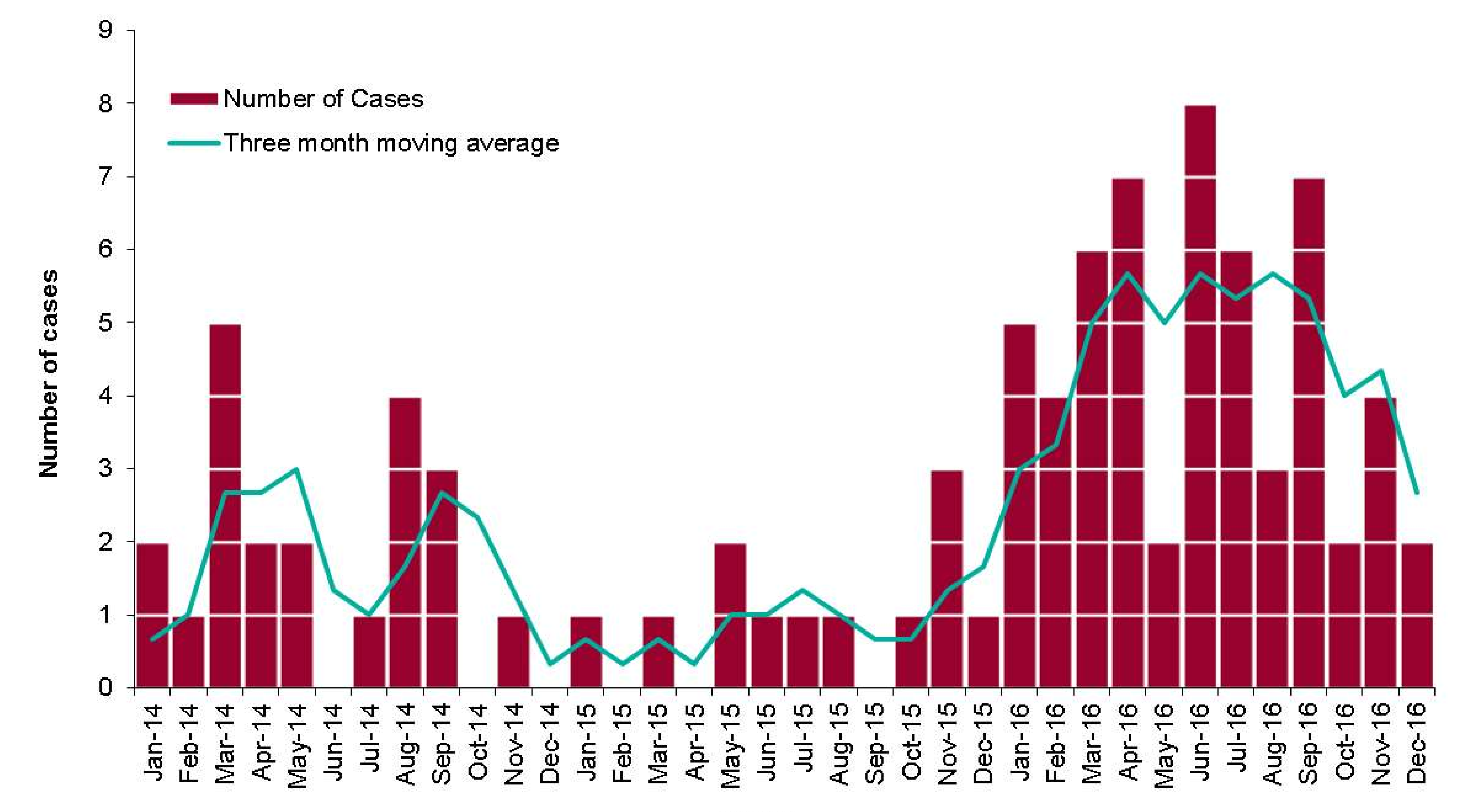

Reasons for attending the clinic varied and included: 27/56 (48\%) attended because they were symptomatic, 16/56 (29\%) cases attending as part of routine STI screening and 13/56 (23\%) for other reasons (contact tracing, GP referral, HIV care, other NHS referral).

Venue and/or web application information was recorded for 40/56 $(71 \%)$ of cases. The most commonly reported venues mentioned by cases were: various bars and clubs in Manchester (7/56, 13\%), various bars and clubs in Birmingham (3/56, $5 \%)$, saunas in Brighton, Middlewich, Manchester and Sandwell (5/56, 9\%), public places in Stoke-on-Trent (4/56, 7\%) and hotels in Gran Canaria $(3 / 55,5 \%)$. Other venues included: a hotel in Benidorm and bars and clubs in London and Liverpool.

GRINDR was the most commonly mentioned application with half $(27 / 56,48 \%)$ of cases reporting use. $3 / 56$ cases $(5 \%)$ mentioned using FabGuys and $3 / 56$ cases (5\%) mentioned using Scruffs. Others mentioned were: BBRT, Boy Ahoy, FitLads, Fab Swingers, Tinder and a Polish website.

Ten of the 56 cases (18\%) reported chemsex use.

Fig 2. Network diagram illustrating venue information/app use by syphilis cases

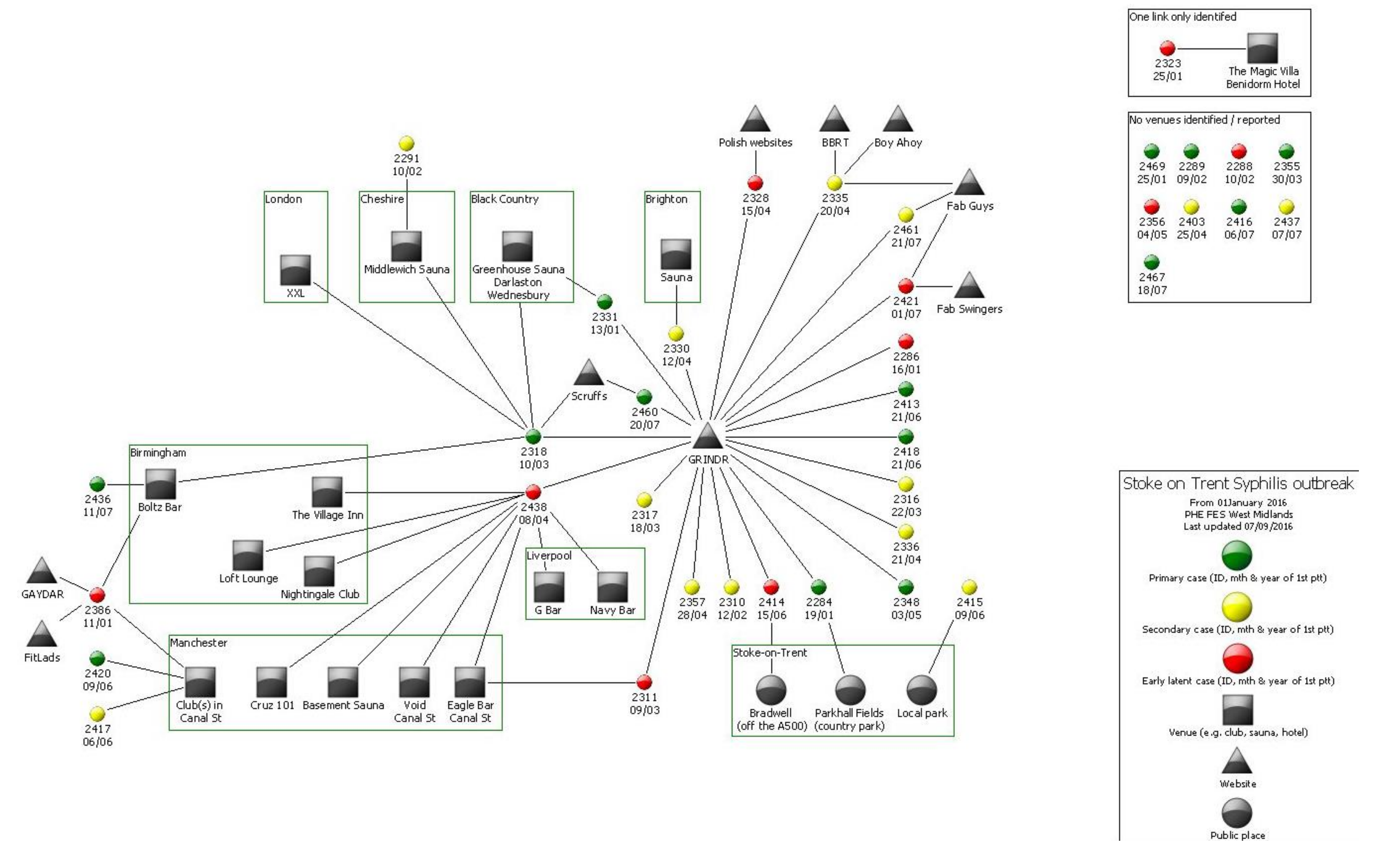

\section{Discussion}

In order to reduce syphilis transmission it is vital that new cases are identified and treated and strategies are put in place to target populations at higher risk. Incident Management Team meetings were held in 2016 with representatives from PHE, GUM, sexual health promotion team and local commissioners. Various control measures were agreed to prevent a further increase in the number of syphilis cases:

- Clinicians at regional sexual health clinics were briefed on the outbreak to ensure rapid identification and treatment of new cases and appropriate education of patients.

- Health promotion messages providing education, information on the outbreak and advice on testing were posted on social network sites (Facebook, Twitter and GRINDR).

- Local GPs were kept updated via our monthly GP newsletter.

- Venues identified through ESSS were made aware of the outbreak and encouraged to engage with health promotion.

- PHE West Midlands engaged with national teams and gained examples from previous outbreaks to share learning.

We continue to monitor cases of infectious syphilis on a monthly basis. 